\title{
Sympathetic Nervous Activity is Involved in the Anti-Inflammatory Effects by Electroacupuncture Stimulation
}

\author{
Byung Gon Jo*, Nam Hoon Kim*, Uk Namgung \\ Department of Korean Medicine, Daejeon University \\ 전침자극 매개성 항염증 반응에 대한 교감신경의 작용연구 \\ 조병곤*(1) - 김남훈*(1) - 남궁욱 ${ }^{(1)}$ \\ 대전대학교 한의학과
}

\begin{abstract}
Objectives : Increasing evidence suggests that parasympathetic vagus nerve activity plays a role in modulating acupunctureinduced anti-inflammatory reaction, but the function of sympathetic nerve is not known. Here, we investigated whether splanchnic sympathetic nerve activity was involved in the regulation of splenic expression of TNF- $\alpha$ mRNA by electroacupuncture (EA) in LPS-injected animals. Methods : DiI was injected into the stomach or celiac ganglion (CG) for retrograde labeling of the target tissues. EA was given at ST36 and the electrical stimulation on the sciatic nerve in LPS-injected mice. c-Fos signals in the tissues were analyzed by immunofluorescence staining, and TNF- $\alpha$ mRNA was analyzed by real-time PCR. Results : Application of EA at ST36 or electrical stimulation on the sciatic nerve induced c-Fos expression in neurons of the spinal cord and celiac ganglion (CG). Then, the vagotomy reduced c-Fos levels in CG neurons but not in the spinal cord in animals given EA. Expression of TNF- $\alpha$ mRNA which was induced in the spleen after LPS was significantly inhibited by EA, then the vagotomy elevated TNF- $\alpha$ mRNA level similar to that in LPS-injected animals. Splanchnectomy in animals given LPS and EA also increased TNF- $\alpha$ mRNA though it was less effective than vagotomy. Conclusions : Our data suggest that EA delivered to the spleen via the splanchnic sympathetic nerve may be involved in attenuating splenic inflammatory responses in LPS-injected animals.
\end{abstract}

Key words : sympathetic nervous system, anti-inflammation, electroacupuncture, TNF- $\alpha$, ST36

\section{Introduction}

Acupuncture therapy has been used for the treatments of diverse body symptoms and diseases in Asian countries and its use becomes expanded into other countries worldwide ${ }^{11}$. However, the traditional theory explaining the action of acupuncture is descriptive, and its scientific basis remains largely elusive. Only recently have experimental studies begun to investigate physiological basis on its action and provide insights into how acupuncture stimulation (AS) could regulate inflammatory reactions in several visceral organs and also in the cardiovascular and central nervous systems ${ }^{2}$. For instance, vagus nerve pathway has been considered as a possible mediator that transmits the acupuncture signals into

Received August 14, 2019, Revised September 17, 2019, Accepted September 17, 2019

Corresponding author: Uk Namgung

Department of Korean Medicine, Daejeon University, Daejeon 34520, Korea

Tel: +82-42-280-2614, Fax: +82-42-274-2600, E-mail: unamgung@dju.kr

*These authors contributed equally to this work.

This research was supported by the Daejeon University fund (Grant number 20180271) (Daejeon, Korea).

(c) This is an open access article distributed under the terms of the Creative Commons Attribution Non-Commercial License (http://creativecommons.org/licenses/ by-nc/4.0) which permits unrestricted non-commercial use, distribution, and reproduction in any medium, provided the original work is properly cited. 
target organs because the efferent vagus nerve activity that might be elicited by descending cerebral signals, somatosensory signals from external environmental, or vagal sensory signals can downregulate the production of inflammatory cytokines in several visceral organs such as spleen, liver, and small intestines ${ }^{3)}$. Indeed, AS was shown to decrease the production of TNF- $\alpha$ in the spleen of LPS-injected animals ${ }^{4}$, and other reports also showed that the vagus nerve activity modulates anti-inflammatory effects of acupuncture in pathologic animal models ${ }^{5-7)}$.

In addition to parasympathetic nerve activity, sympathetic nerve activity may be involved in the regulatory process of cellular uptake of glucose, gastrointestinal and rectal motility, cardiovascular baroreceptor reflex, and heart rate variability by $\mathrm{AS}^{8-10)}$. In neuroanatomical point of view, sensory modalities caused by cutaneous AS transmit nerve signals into the spinal ascending pathway and further up to the several brain areas including cerebral cortex. Somatosensory stimulation including AS can relay synaptic inputs into neurons in the nucleus tractus of solitaries (NTS) and the rostral ventrolateral medulla (RVLM) that are connected to the vagal pathway and also, through the descending neural pathway, to spinal preganglionic sympathetic neurons and thus may regulate cardiovascular function via sympathetic nerve pathway, ${ }^{2,11-13)}$. Furthermore, noradrenergic neurotransmitters induce neuroimmune modulation through the activation of iNKT cells, promoting inflammation and immunosuppression ${ }^{14}$. Allen and colleagues claimed that electrical stimulation of the splanchnic sympathetic nerve connected to the celiac ganglion (CG) attenuated splenic inflammatory responses in LPS-injected animals ${ }^{15}$. Collectively, it may be possible that AS, once transmitted into the sympathetic nerve pathway in the central nervous system, participates in neuroimmune interaction for the regulation of inflammatory responses in target organs.

Here, using LPS-induced endotoxemic rat model, we explored whether the splanchnic nerve pathway connected to CG is related to EA-mediated regulation of splenic expression of TNF- $\alpha$ mRNA. Our data provide evidence that sympathetic nerve activity contributes to anti-inflammatory responses by AS.

\section{Materials and Methods}

\section{Experimental animals}

ICR mice ( 7 weeks old male, 20 25 g, Samtako) were maintained in an animal room with regulated temperature $\left(22^{\circ} \mathrm{C}\right)$, 60\% humidity, and a 12-hour light/12-hour dark cycle. Animals were allowed to eat commercial chow and to drink water ad libitum. All protocols involving preopertative, operative, and postoperative animal care were approved by the Daejeon University Institutional Animal Use and Care Committee (Daejeon, Korea) and were in accordance with the Animal-use Statement and Ethics Committee Approval Statement for Animal Experiments provided by Daejeon University (Approval number: DJUARB2019-025; Daejeon, Korea). None of animals became ill and died during the study. Postoperative animals were euthanized by overdose of ketamine (80 $\mathrm{mg} / \mathrm{kg}$ ) and zylazine $(5 \mathrm{mg} / \mathrm{kg})$. All surgical procedures were conducted in a room under well-ventilated, sterile conditions, and all efforts were made to minimize suffering.

\section{Retrograde tracing}

To anatomically identify CG, we injected DiI (3 $\mu 1$ of $0.5 \%$ in DMSO; Sigma-Aldrich) in cardiac orifice and pylorus and prepared CG 3 days later. CG sections (10 $\mu \mathrm{m}$ thickness) were prepared and incubated with Hoechst $33258(25 \mu \mathrm{g} / \mathrm{ml}$, bis-benzimide; Sigma-Aldrich) for $10 \mathrm{~min}$. DiI-labeled neurons in the CG were analyzed under the fluorescence microscope. Once we confirmed anatomical location of CG in the abdomen, we injected DiI ( $3 \mu 1$ of $0.5 \%$ in DMSO) into the CG, prepared the spleen and spinal cord at thoracic levels $6 \sim 9$, and analyzed DiI signaling by using fluorescence microscope.

\section{Endotoxemia and animal surgery}

To induce endotoxemia, animals were i.p. injected with lipopolysaccharide (LPS; 15 mg/kg; Sigma-Aldrich). For vagotomy, mice were anesthetized with ketamine and xylazine and the left vagus nerve was exposed on a cervical midline and cut with scissors. For splanchnectomy (SPNX), splanchnic nerve connected to CG was exposed and rapidly cut. Animals after surgeries were quickly sutured and recovered 
from narcosis. Animals after surgery were recovered and used for the experiments 7 days later.

\section{Electroacupuncture stimulation \& sciatic nerve stimulation}

Prior to EA administration, mice were anesthetized by i.p. injection of ketamine $(80 \mathrm{mg} / \mathrm{kg})$ and xylazine $(5 \mathrm{mg} / \mathrm{kg})$. Acupuncture needle $(0.20 \times 7 \mathrm{~mm}$, HL Medical, Seoul, Korea) was inserted bilaterally into ST36 (3 4 mm below the knee midline and laterally $1 \sim 2 \mathrm{~mm}$ at a depth of $2 \sim 3 \mathrm{~mm}$ ), as has been used in our previous study ${ }^{4)}$. Acupuncture needle was inserted for 30 minutes with a slow rotation every 5 minutes. The electric stimulation was given at $1 \mathrm{~V}, 1 \mathrm{~Hz}$, with a $2 \mathrm{~ms}$ of pulse duration for $30 \mathrm{~min}$ by using an isolated pulse stimulator (Isolated pulse stimulator model 2100, A-M Systems, Sequim, WA, USA). For sciatic nerve stimulation (SNS), sciatic nerves were exposed bilaterally by incising the middle of the hind leg in anesthetized mice and a bipolar hook electrode of nichrome wire was placed on the nerve. The electrical current $(10 \mathrm{~mA}, 5 \mathrm{~Hz}$ with $5 \mathrm{~ms}$ of pulse duration) was delivered for $5 \mathrm{~min}$ by using a stimulator (Isolated pulse stimulator model 2100, A-M Systems). Animals were sacrificed $2 \mathrm{~h}$ after EA or SNS. Tissues such as spinal cord at thoracic levels $6 \sim 9, C G$, and spleen were prepared and used for real-time PCR for TNF- $\alpha$.

\section{Immunofluorescence staining}

Spleen, spinal cord and CG tissues were embedded and frozen at $-20^{\circ} \mathrm{C}$. Sections (15 thickness) of spinal cord, CG and spleen were cut on a cryostat, mounted on positively charged slides, and subjected to immunofluorescence staining. Sections were treated with fixation buffer ( $4 \%$ paraformaldehyde and 4\% sucrose in 1X PBS) at room temperature for $45 \mathrm{~min}$, washed 3 times for $5 \mathrm{~min}$ with glycine, $10 \mathrm{mM}$ in PBST (1X PBS and $0.1 \%$ triton X-100), and permeabilized with $0.5 \% \mathrm{NP}-40$ in PBS at room temperature for $30 \mathrm{~min}$. Then, the sections were treated with blocking solution (5\% horse serum and $5 \%$ bovine serum albumin in PBST) for overnight at $4^{\circ} \mathrm{C}$, incubated $4 \mathrm{~h}$ at room temperature with primary antibodies, and washed three times for 10 min each with
PBST. Sections were incubated with secondary antibodies for $2 \mathrm{~h}$ at room temperature in a dark room and washed three times with PBST. Cellular nuclei were stained with Hoechst 33258 pentahydrate $(25 \mu \mathrm{g} / \mathrm{ml}$, bis-benzimide; Sigma-Aldrich, St. Louis, MO, USA) for $10 \mathrm{~min}$ before the final wash with PBST. Fluorescence images were analyzed with a Nikon fluorescence microscope, and selected images were captured and further analyzed by Photoshop program. Primary antibodies used in the present study were anti-c-Fos (Rabbitpolyclonal, 1:400, Santa Cruz Biotech, Dallas, TX, USA) and anti-neurofilament 200 (NF-200) (Mouse-monoclonal, 1:400, Sigma-Aldrich) antibodies. Secondary antibodies were antifluorescein-goat anti-mouse IgG (1:400, Molecular probes, Eugene, OR) and anti-rhodamine-goat anti-rabbit IgG antibodies (1:400, Molecular probes). For quantification of c-Fos signals in sections of spinal cord, CG, and spleen, fluorescence images in the slide were captured by fluorescence microscope coupled with digital camera and transferred to the i-Solution software program (Image and Microscope Technology, Daejeon, Korea). Digital images for analysis were taken under the same exposure conditions among experimental groups and no modifications in the brightness and contrast were made for the numerical measures of pixel intensity.

\section{Real-time PCR}

RNA was isolated from the spleen by using Easy-BLUE reagent (Intron, Seongnam, Korea). cDNA synthesis was performed using $1 \mu \mathrm{M}$ reaction buffer $(50 \mathrm{mM}$ Tris- $\mathrm{HCl}, 75 \mathrm{mM}$ $\mathrm{KCl}, 3 \mathrm{mM} \mathrm{MgCl}$ 2, $10 \mathrm{mM}$ DTT), $104 \mu \mathrm{M}$ dNTP, RNasin (30 $\mathrm{U})$, random primers (16 $\mu \mathrm{M}$, Promega, Madison, WI), MMLV reverse transcriptase $(200 \mathrm{U})$ for 2 hours at $37^{\circ} \mathrm{C}$ in an incubator. Real-time PCR was carried out in a $20 \mu 1$ of reaction containing synthetized cDNA $(3 \mu \mathrm{g}), 1 \mathrm{X}$ Power SYBR Green PCR Master mix (Life Technologies, Carlsbad, CA), $0.15 \mu \mathrm{M}$ of forward and reverse primers of TNF- $\alpha$ mRNA (5'-CATCTTCTCAAAATTCGAGTGACAA-3' and 5'-TG GGAGTAGACAAGGTACAACCC-3' respectively) in each well of 96-well reaction plate (MicroAmp Optical, Applied Biosystems, Waltham, MA, USA). Real-time PCR for GAPDH 
reference mRNA synthesis was done in a similar reaction condition by using GAPDH primer (Mouse GAPD, Applied Biosystems) and endogenous control (VIC1/MGB probe primer). Plate was covered with a film (MicroAmp Optical Adhesive Film, Applied Biosystems) and centrifuged to spin down the sample. Reaction was carried out using an 7500 Real Time PCR System (Applied Biosystems) by activating Taq polymerase for $2 \mathrm{~min}$ at $50^{\circ} \mathrm{C}$ and for $10 \mathrm{~min}$ at $95^{\circ} \mathrm{C}$, followed by 40 cycles with $15 \mathrm{~s}$ at $95^{\circ} \mathrm{C}$ (denaturation) and $1 \mathrm{~min}$ at $60^{\circ} \mathrm{C}$ (annealing). The results were shown as a fold change of TNF- $\alpha$ mRNA to GADPH reference mRNA. The relative quantification (RQ) value of TNF- $\alpha$ mRNA expression to GAPDH reference mRNA was calculated by the threshold cycle $(\mathrm{Ct})$ data.

\section{Statistical analysis}

Data were presented as standard error of mean (SEM). The means of the data in individual groups were compared by using the one-way ANOVA with Tukey's post-hoc tests for multiple comparisons (GraphPad Prism 7). Statistically significant differences were reported as ${ }^{*} p<0.05,{ }^{* * *} p<0.01$, or ****: $p<0.001$.

\section{Results}

In order to identify the anatomical location of $C G$ and its neuronal connectivity to the spleen, we injected DiI into stomach and analyzed retrogradely labeled neurons in the CG. Red-fluorescence-labeled neurons were observed mostly in the peripheral zone of $C G$ in mice 3 days after DiI injection in the cardiac orifice and pylorus (marked arrows in Fig. 1A). To further investigate the connection of CG to the spinal cord and spleen as preganglionic inputs into and postganglionic targets of CG respectively, we injected DiI into the CG and examined DiI signals in the spleen and spinal cord. DiIsignals were detected in the splenic hilum area (Fig. 1B) and in the spinal cord (Fig. 1C). It was noted that the DiI signals
A
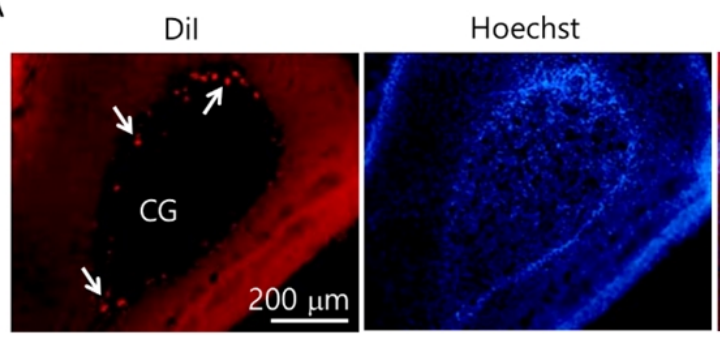

C

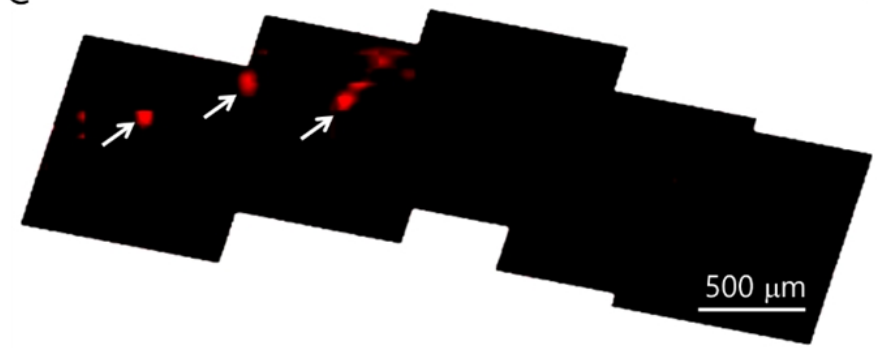

merge

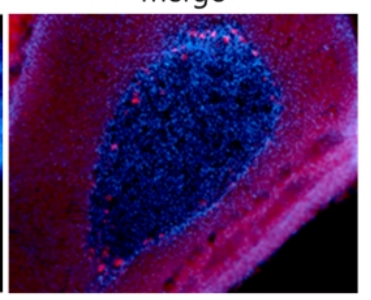

D
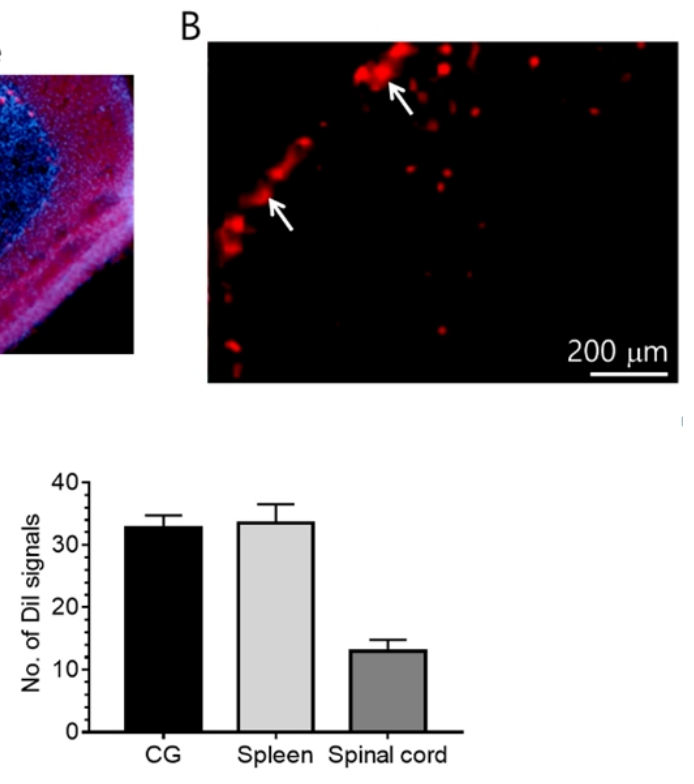

Fig. 1. Identification of sympathetic nerve pathway connected to celiac ganglion (CG) and spleen by Dil tracing.

(A) Detection of CG neurons that have connections to stomach. Dil was injected into the cardia and pylorus of stomach in mice, and 3 days later, CG sections were prepared for fluorescence image analysis. In (B) and (C), Dil was injected into the CG, and 3 days later, sections from the spleen and the spinal cord (thoracic level 6 9) were prepared for the detection of Dil signals, respectively. (B) Fluorescence view of Dil in the splenic hilum sections. (C) Dil signals in spinal cord sections. Multiple images in the spinal cord sections at lower thoracic levels 6 9 are aligned into a single image. (D) Quantification of Dil-labeled individual neurons in the CG, spleen and spinal cord after the injection of Dil into the corresponding locations as indicated in (A) (C) above. Mean \pm SEM, $N($ Number of independent experiments) $=4$. 
were aligned parallel to the intermediolateral (IML) zone in the spinal cord where the sympathetic neuronal cell bodies are located. Quantitative measurements of DiI-labeled neurons in the CG, spleen, and spinal cord showed similar levels of signals in the CG and spleen, and lower signals in the spinal cord (Fig. 1D).

To investigate whether EA induces the activation of sympathetic neurons, we applied EA at ST36 and analyzed neuronal responses in the spinal cord and the CG. Unlike the spinal cord sections from the untreated control animals, c-Fos signals were observed by EA or SNS in the spinal cord at thoracic levels 6 9 (Fig. 2). To further examine whether vagus nerve activity was involved in neuronal activation in the spinal cord, we measured c-Fos after vagotomy. c-Fos signals were detected after EA and SNS in vagotomized animals (VNX), without showing distinct changes in its intensity from EA and SNS groups.

We further investigated the effects of EA on c-Fos induction in the CG. As shown in Fig. 3A, c-Fos signals were detected in the CG after EA and SNS, but the signal intensity was decreased after VNX (Fig. 3A). Quantitative measurement revealed that levels of c-Fos signal in EA and SNS groups were significantly higher than control group. Then, c-Fos levels in EA+VNX and SNS+VNX were significantly decreased compared to the corresponding EA and SNS groups although c-Fos signal intensity in EA+VNX and SNS+VNX was obviously stronger than that in the control group (Fig. 3B).

We next sought to evaluate the regulatory effects of EA on LPS-induced inflammation in the spleen. Real-time PCR analysis showed that mRNA levels of inflammatory cytokine $\mathrm{TNF}-\alpha$ was markedly increased in the spleen after LPS (Fig. 4). However, the expression level was significantly decreased by EA, close to that of untreated control animals. After the vagotomy (VNX), TNF- $\alpha$ mRNA level was similar to that of LPS group, but still was significantly higher than that of LPS+EA animals. We further found that SPNX significantly increased TNF- $\alpha$ level compared to LPS+EA animals. TNF- $\alpha$ levels in LPS+EA+VNX and LPS+EA+SPNX groups reached 87 and $65 \%$ to that of LPS animals, respectively, thus suggesting that VNX and SPNX were both effective in abrogating antiinflammatory activity of EA in LPS animals.

\section{Discussion}

Growing body of evidence indicate that the nerve activity plays a role in modulating acupuncture therapy for the regulation of internal organ diseases and pain and the vagus nerve has been particularly considered as a potential mediator transmitting acupuncture signals into the target organs ${ }^{2,16,17)}$. Given that the vagus nerve stimulation has been identified as inducing inhibitory signaling pathway for the production of anti-inflammatory cytokines including $\mathrm{TNF}-\alpha^{3}$, it is tempting to speculate that the vagus nerve activity triggered by AS may regulate inflammatory diseases ${ }^{4,6,18)}$. However, there has been no mechanistic study examining whether AS regulates the inflammatory response through sympathetic pathways. Here, we found that AS activates sympathetic neural pathway
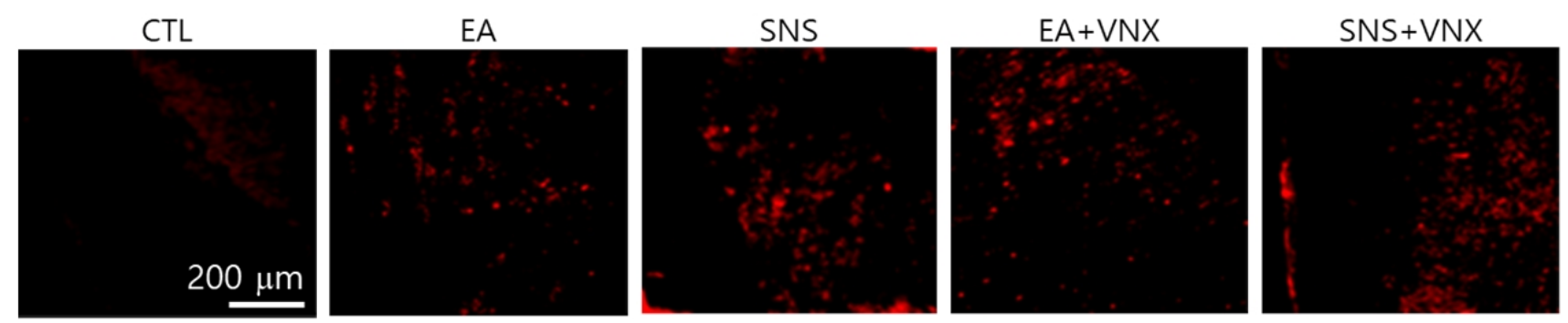

Fig. 2. Identification of c-Fos signals in the spinal cord after EA, sciatic nerve stimulation (SNS) and vagotomy (VNX).

Spinal cord sections at thoracic levels $6 \sim 9$ were prepared from mice $2 \mathrm{~h}$ after EA and SNS and used for immunofluorescence staining for c-Fos protein (red). Detailed descriptions on experimental treatments of EA, SNS and VNX are described in the Materials and Methods sections. 
A
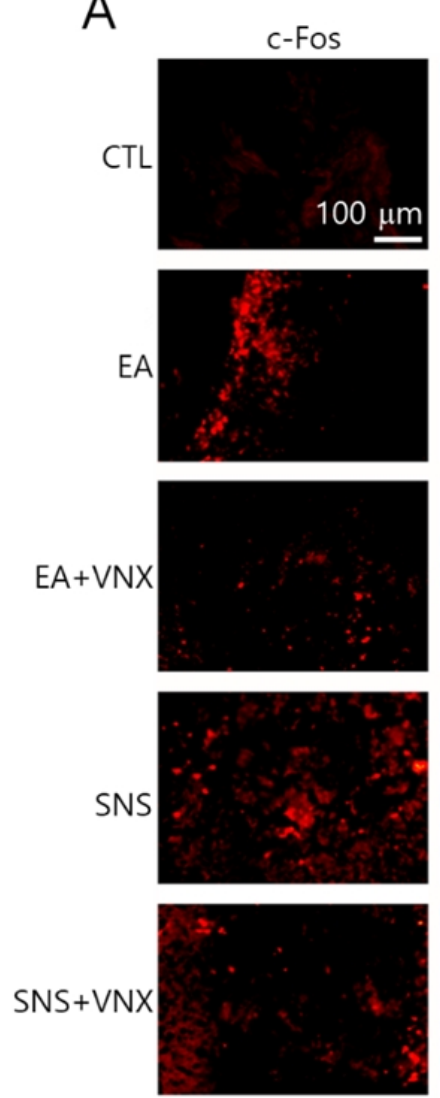

Hoechst
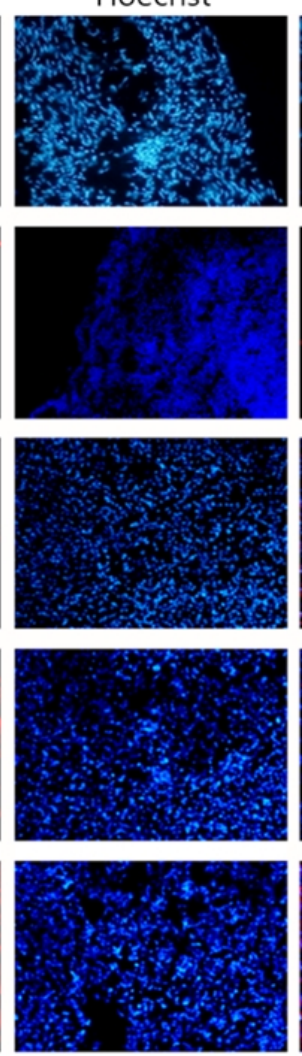

c-Fos/Heochst
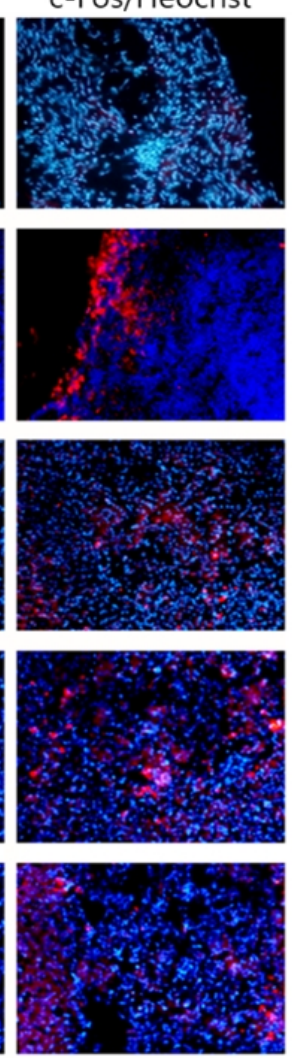

B



Fig. 3. Detection of c-Fos signals in the CG after EA, sciatic nerve stimulation (SNS) and vagotomy (VNX) in mice.

CG were prepared from mice which had undergone treatments such as EA, SNS and VNX as described in the Materials and Methods. (A) Representative images of immunofluorescence staining of CG sections. (B) A plot for quantification of c-Fos signal in the CG sections. Average value of the signals in the sections of individual animals were compared among experimental groups. Mean \pm SEM, Number of animals per each group $=4$. ${ }^{\star \star *} p<0.001$ (one-way ANOVA).

including the CG and the spleen as its target and attenuates splenic inflammation in an animal model of endotoxemia.

Splanchnic nerve arising from IML sympathetic neurons in lower thoracic spinal cord is connected to neurons in the CG, and postganglionic efferent fibers innervate cells in the target organs such as stomach, liver, pancreas, and spleen ${ }^{19)}$. Here in the present study, we have confirmed the connections of the pre- and postganglionic sympathetic nerve fibers to CG by using retrograde tracing technique and then analyzed the responses of IML neurons in the spinal cord after AS. AS at ST36 was shown to induce neuronal response in the dorsal root ganglion at levels of lumbar $4 \sim 6^{4,20)}$, which would transmit signals into the spinal cord and further up to the specific brain areas. Our data show that both EA at ST36 and SNS induced c-Fos signals in the IML area, suggesting the signaling connection between ascending spinal pathway of DRG neurons and IML sympathetic neurons, possibly via descending brain stem pathway ${ }^{11)}$. Somatosensory afferent nerve fibers have synaptic relays onto neurons in the RVLM, which are in turn connected to spinal sympathetic neurons $^{12,13)}$ (see Fig. 5). Moreover, several studies reported that acupuncture stimulation are involved in regulating sympathetic nerve activity such as gastric motility, rectal motility, and cardiovascular baroreceptor reflex ${ }^{8,9,13)}$. Future studies would be critical to define signaling events between neurons in the ascending somatosensory pathway bearing the information of acupuncture sensory modalities and spinal sympathetic neurons. When we disconnected vagus nerve at cervical level, c-Fos signals in the spinal cord were not abated, implying that vagus nerve activity which could 


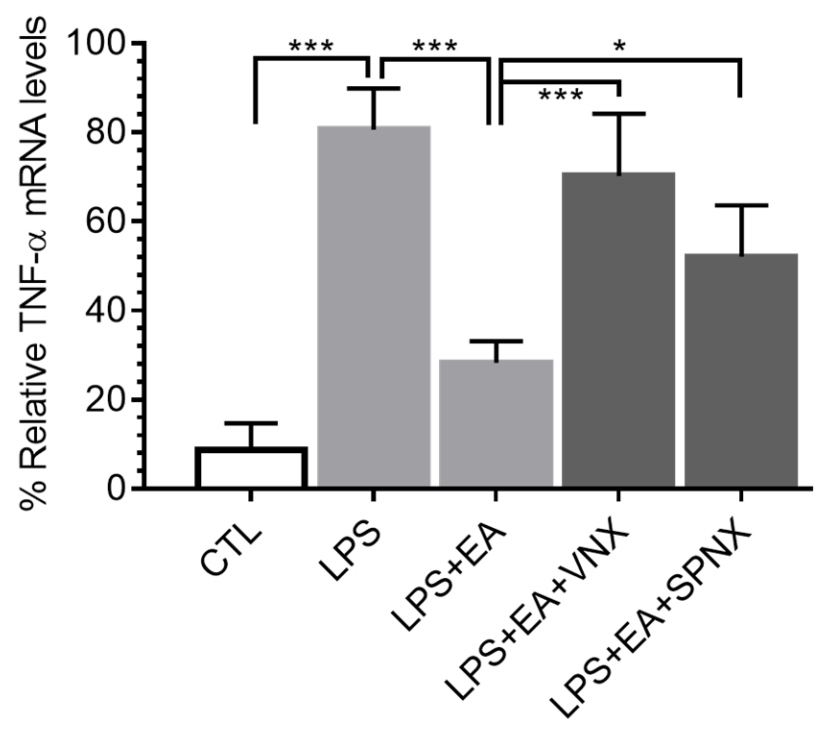

Fig. 4. Regulation of TNF- $\alpha$ mRNA expression in the spleen by $\mathrm{EA}$, sciatic nerve stimulation (SNS) and vagotomy (VNX).

EA was given $30 \mathrm{~min}$ after LPS injection and animals were sacrificed $2 \mathrm{~h}$ later. Splenic RNA was extracted from mice after different treatment as indicated in the figure and used for realtime PCR. Levels of TNF- $\alpha$ mRNA relative to GAPDH mRNA were plotted and compared among experimental groups. Mean \pm SEM, Number of animals per group $=4{ }^{\star} p<0.05$, and ${ }^{* \star \star} p<0.001$ (oneway ANOVA).

be induced by AS did not affect the signal transmission through the splanchnic sympathetic pathway.

Our data further showed that EA induced neuronal responses in the CG in terms of c-Fos expression. Interestingly however, levels of c-Fos expression induced by EA and SNS were weakened by vagotomy. Recent studies have revealed that the vagus nerve destined to innervate spleen has efferent nerve connections to $\mathrm{CG}$ and then cholinergic inputs into splenic macrophages via $\mathrm{T}$ lymphocytes ${ }^{21)}$. Thus, c-Fos expression in CG cells in response to AS may be caused by combined vagal and splanchnic nerve activities, as shown in Fig. 5.

We have previously reported that AS decreases the splenic production of TNF- $\alpha$ through the vagal modulation in LPSinjected animals ${ }^{4}$. Here, the present data, while confirming anti-inflammatory effects of EA in the spleen, provide new evidence on the involvement of sympathetic nerve activity. Levels of TNF- $\alpha$ mRNA were decreased to $35 \%$ in LPS+EA animals compared to LPS animals, then elevated to $87 \%$ and $65 \%$ of that of LPS group after VNX and SPNX, respectively,

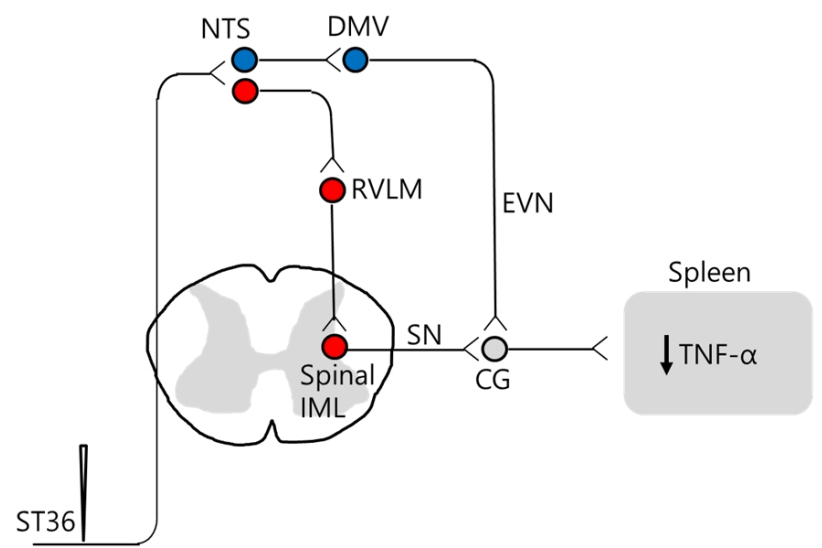

Fig. 5. A hypothetical scheme illustrating the neural pathways leading to splenic anti-inflammation after EA at ST36.

EA at ST36 may transmit the signals either into vagal pathway (neuronal cell bodies marked in blue) or into spinal sympathetic pathway (neuronal cell bodies marked in red), converge into CG neurons, and regulate the production of TNF- $\alpha$ in the spleen. NTS : nucleus tractus solitaries, DMV : dorsal motor nucleus of the vagus nerve, RVLM : rostral ventrolateral medulla, CG : celiac ganglion, IML : intermediolateral, SN : splanchnic nerve, EVN : efferent vagus nerve.

highlighting the contribution of EA-induced sympathetic nerve activity to anti-inflammation. This notion is consistent with immunofluorescence data revealing that c-Fos signals in the CG were downregulated by VNX. Both vagal and splanchnic sympathetic nerve activities are converged into neurons in the CG and may contribute to anti-inflammatory signaling events in the spleen through the activation of $\mathrm{T}$ lymphocytes. Accordingly, neural signals arising from EA at ST36 may be transmitted through both vagal and sympathetic pathway which are both converged into the CG and regulate inflammatory responses in the spleen (Fig. 5).

Adrenergic nerve activity was shown to be transmitted into the liver and regulate inflammatory response through hepatic iNKT cells ${ }^{14)}$. Whether or not similar neuroimmune communication mechanisms are involved in the sympathetic regulation for anti-inflammatory regulation in the spleen remains to be explored ${ }^{22}$. Finally, McAllen and colleagues have claimed that the splanchnic sympathetic nerve activity is primarily responsible for cholinergic anti-inflammatory reaction in the spleen and the vagus nerve activity would not be involved in this process because they could not identify vagus nerve activity transmitted into the $C G^{15)}$. Further 


\section{KOREAN JOURNAL OF ACUPUNCTURE}

anatomical and physiological studies will be critical to clarify the characteristics of vagus nerve activity innervating major internal organs such as spleen, liver, intestines and others and to further explore the biological basis of acupuncture therapy for the treatment of diseases ${ }^{23}$. It should also be mentioned that in the present study, we examined the regulation of TNF- $\alpha$ only as an indicator of inflammatory response. We found that the electrical stimulation of the vagus nerve attenuated hepatic production of IL-1 $\beta$ and IL-6 in addition to TNF- $\alpha$ (unpublished data). Thus, further studies examining the regulation of other inflammatory cytokines by AS will be critical to confirm the present findings. Also, quantitative assessment of neuronal activation (e.g., levels of postsynaptic membrane receptors) in the spinal cord and in the CG in responses to AS will strengthen the concept of the neural basis of acupuncture. In conclusion, our provide evidence for the first time that EA can regulate inflammatory reaction in the spleen via splanchnic-CG pathway and thus imply that EA-induced sympathetic nerve activity may play a role in inflammatory disease of visceral organs and systemic inflammation.

\section{Acknowledgement}

This research was supported by the Daejeon University fund (Grant number 20180271) (Daejeon, Korea).

\section{Conflicts of interest}

The authors have declared that no conflicts of interest exist.

\section{References}

1. Leake R, Broderick JE. Treatment of efficacy of acupuncture: a review of the research literature. Integrative Med. 1999 ; 1 : 107-15.
Sympathetic regulation of electroacupuncture effects

2. Park JY, Namgung U. Electroacupuncture therapy in inflammation regulation: current perspectives. J Inflamm Res. 2018 ; 11 : 227-37. https://doi.org/10.2147/JIR.S141198.

3. Tracey KJ. The inflammatory reflex. Nature. 2002 ; 420(6917) : 853-9. https://doi.org/10.2147/JIR.S141198.

4. Lim HD, Kim MH, Lee CY, Namgung U. Anti-Inflammatory Effects of Acupuncture Stimulation via the Vagus Nerve. PLoS One. 2016 ; 11(3) : e0151882. https://doi.org/10.1371/journal. pone.0151882.

5. Jin H, Guo J, Liu J, Lyu B, Foreman RD, Yin J, et al. Anti-inflammatory effects and mechanisms of vagal nerve stimulation combined with electroacupuncture in a rodent model of TNBS-induced colitis. Am J Physiol Gastrointest Liver Physiol. 2017 ; 313(3) : G192-202. https://doi.org/10.1152/ajpgi.00254. 2016.

6. Torres-Rosas R, Yehia G, Peña G, Mishra P, del Rocio ThompsonBonilla M, Moreno-Eutimio MA, et al. Dopamine mediates vagal modulation of the immune system by electroacupuncture. Nat Med. 2014 ; 20(3) : 291-5. https://doi.org/10.1038/nm.3479.

7. Du MH, Luo HM, Hu S, Lv Y, Lin ZL, Ma L. Electroacupuncture improves gut barrier dysfunction in prolonged hemorrhagic shock rats through vagus anti-inflammatory mechanism. World J Gastroenterol. 2013 ; 19(36) : 5988-99. https://doi.org/10. 3748/wjg.v19.i36.5988.

8. Chen J, Song GQ, Yin J, Koothan T, Chen JD. Electroacupuncture improves impaired gastric motility and slow waves induced by rectal distension in dogs. Am J Physiol Gastrointest Liver Physiol. 2008 ; 295(3) : G614-20. https://doi.org/10.1152/ ajpgi.90322.2008.

9. Yu Z, Zhang N, Lu CX, Pang TT, Wang KY, Jiang JF, et al. Electroacupuncture at ST25 inhibits jejunal motility: Role of sympathetic pathways and TRPV1. World J Gastroenterol. 2016; 22(5) : 1834-43. https://doi.org/10.3748/wjg.v22.i5.1834.

10. Benrick A, Kokosar M, Hu M, Larsson M, Maliqueo M, Marcondes $\mathrm{RR}$, et al. Autonomic nervous system activation mediates the increase in whole-body glucose uptake in response to electroacupuncture. FASEB J. 2017 ; 31(8) : 3288-97. https://doi.org/ 10.1096/fj.201601381R.

11. Potts JT, Paton JF, Mitchell JH, Garry MG, Kline G, Anguelov PT, et al. Contraction-sensitive skeletal muscle afferents inhibit ar- 
terial baroreceptor signalling in the nucleus of the solitary tract: role of intrinsic GABA interneurons. Neuroscience. 2003 ; 119(1) : 201-14. https://doi.org/10.1016/s0306-4522(02)00953-3.

12. Zagon A, Smith AD. Monosynaptic projections from the rostral ventrolateral medulla oblongata to identified sympathetic preganglionic neurons. Neuroscience. 1993 ; $54(3)$ : 729-43. https:// doi.org/10.1016/0306-4522(93)90243-9.

13. Tjen-A-Looi SC, Guo ZL, Fu LW, Longhurst JC. Paraventricular Nucleus Modulates Excitatory Cardiovascular Reflexes during Electroacupuncture. Sci Rep. 2016 ; 6 : 25910. https://doi.org/ 10.1038/srep25910.

14. Wong CH, Jenne CN, Lee WY, Léger C, Kubes P. Functional innervation of hepatic iNKT cells is immunosuppressive following stroke. Science. 2011 ; 334(6052) : 101-5. https://doi.org/ 10.1126/science.1210301.

15. Martelli D, Yao ST, McKinley MJ, McAllen RM. Reflex control of inflammation by sympathetic nerves, not the vagus. J Physiol. 2014; 592(7) : 1677-86. https://doi.org/ 10.1113/jphysiol.2013. 268573.

16. Zhao ZQ. Neural mechanism underlying acupuncture analgesia. Prog Neurobiol. 2008; 85(4) : 355-75. https://doi.org/ 10.1016/ j.pneurobio.2008.05.004.

17. Kavoussi B, Ross BE. The neuroimmune basis of anti-inflammatory acupuncture. Integr Cancer Ther. 2007 ; 6(3) :
251-7. https://doi.org/10.1177/1534735407305892.

18. Song JG, Li HH, Cao YF, Lv X, Zhang P, Li YS, et al. Electroacupuncture improves survival in rats with lethal endotoxemia via the autonomic nervous system. Anesthesiology. 2012 ; 116(2) : 406-14. https://doi.org/10.1097/ALN. 0b013 e3182426ebd.

19. Berthoud HR, Neuhuber WL. Functional and chemical anatomy of the afferent vagal system. Auton Neurosci. $2000 ; 85: 1-17$. https://doi.org/10.1016/S1566-0702(00)00215-0.

20. Kim MH, Park YC, Namgung U. Acupuncture-stimulated activation of sensory neurons. J Acupunct Meridian Stud. 2012 ; 5(4) : 148-55. https://doi.org/10.1016/j.jams.2012.05.002.

21. Rosas-Ballina M, Ochani M, Parrish WR, Ochani K, Harris YT, Huston JM, et al. Splenic nerve is required for cholinergic antiinflammatory pathway control of TNF in endotoxemia. Proc Natl Acad Sci USA. 2008 ; 105: 11008. https://doi.org/10.1073/ pnas.0803237105.

22. Bellinger DL, Felten SY, Lorton D, Felten DL. Origin of noradrenergic innervation of the spleen in rats. Brain Behav Immun. $1989 ; 3: 291-311$.

23. Willemze RA, Luyer MD, Buurman WA, de Jonge WJ. Neural reflex pathways in intestinal inflammation: hypotheses to viable therapy. Nat Rev Gastroenterol Hepatol. 2015 ; 12(6): 353-62. https://doi.org/10.1038/nrgastro.2015.56. 